Review

\title{
Role of MicroRNA-30c in cancer progression
}

\author{
Wenyan $\operatorname{Han}^{1 *}$, Hongwei Cui ${ }^{*}$, Junqing Liang ${ }^{3}$ and Xiulan $\mathrm{Su}^{2 \bowtie}$ \\ 1. Laboratory of the Second Affiliated Hospital of Inner Mongolia Medical University.No.1 Yingfang Road, Huimin District, Hohhot, Inner Mongolia, China. \\ 2. Clinical Medical Research Center of the Affiliated Hospital/Inner Mongolia Key Laboratory of Medical Cellular Biology, Inner Mongolia Medical \\ University, Hohhot, 010050, Inner Mongolia, P.R. China \\ 3. Department of Breast Oncology, Inner Mongolia Autonomous Region Cancer Hospital, Hohhot, 010000, Inner Mongolia, P.R. China \\ ${ }^{*}$ Co-first authors \\ $\square$ Corresponding author: Xiulan Su, Clinical Medical Research Center of the Affiliated Hospital of Inner Mongolia Medical University, Tongdao North Stress \\ No. 1, Hohhot, Inner Mongolia, 010050, People's Republic of China. Tel: +86 13904710692; Fax: +86 471 3451709; E-mail: xlsu@hotmail.com. \\ (c) The author(s). This is an open access article distributed under the terms of the Creative Commons Attribution License (https://creativecommons.org/licenses/by/4.0/). \\ See http://ivyspring.com/terms for full terms and conditions.
}

Received: 2019.07.16; Accepted: 2020.01.21; Published: 2020.02.14

\begin{abstract}
MicroRNAs (miRNAs or miRs) is a non-coding small RNA of a type of 18 24 nucleotide-regulated gene that has been discovered in recent years. It mainly degrades the target gene mRNA or inhibits its translation process through the complete or incomplete bindings with 3'UTR of target genes, followed by the regulation of individual development, apoptosis, proliferation, differentiation and other life activities through the post-transcriptional regulation. Among many miRNAs, the microRNA family, miR-30, plays diverse roles in these key process of neoplastic transformation, metastasis, and clinical outcomes in different cancer progression. As key member of miR-30, miR-30c is regulated by oncogenic transcription factors and cancer progression related genes. Recently, numerous studies have demonstrated that the aberrant expression of miR-30c was significantly associated with the majority of human cancer progression. In this review, the diverse roles of miR-30c in different cancer progression such as the cellular and molecular mechanisms, the potential applications in clinics were summarized to speculate the benefits of miR-30c over-expression in cancer treatment and prognosis.
\end{abstract}

Key words: microRNAs, microRNA-30c, cancer progression, cancer treatment, cancer prognosis

\section{Introduction}

MicroRNAs (miRNAs or miRs)are endogenous, non-coding single-stranded small-molecule RNAs of a class of 18 to 24 nucleotide-regulated genes. The specific bindings between miRNAs and the $3^{\prime}$ untranslated region ( $\left.3^{\prime} \mathrm{UTR}\right)$ of the target mRNA lead to the degradation or translational inhibition of the target mRNA, which results in post-transcriptional gene silencing. The specific associations between miRNAs and target mRNA depends on the complementarities between miRNAs and the target mRNAs. The degradation of target mRNA occurs when complementarities is complete or nearly complete, which is more common in plant miRNAs, while most mammalian miRNAs inhibit the post-transcriptional translation of target mRNA by the formation of incomplete complementary sequence $[1,2]$.

In human beings, the majority of miRNAs are located in introns or non-coding mRNA transcripts of protein-coding genes, while the remaining miRNAs are distant from the transcripts in the genome, such as the exons of non-coding mRNA genes, or 3' UTR of mRNA genes, or other miRNAs genes[3]. In recent years, more and more studies have proved that miRNAs play important roles in a variety of cancer progression, such as respiratory cancers[4], digestive system cancers[5,6], neurological system cancers[7], genitourinary cancers[8] and vascular cancers[9]. miRNAs regulate the proliferation, differentiation, invasion, apoptosis and metastasis process of cancer cells in the form of oncogenes or tumor suppressor genes[10,11].It is worth noting that miRNAs and their target mRNA play synergistic network roles in cancer progression, and the analyses of miRNA expressions have identified potential markers for the diagnosis, prognosis and treatment of cancer patients[12,13].In these respects, more and more studies have confirmed 
that microRNA-30 family members maintain low expression levels in different cancer tissues and play a tumor suppressor role in cancer progression, metastasis and drug resistance[14,15].In this review, the role of microRNA-30c (miR-30c) in cancer progression was summarized to address the consequences associated with reduced expression levels of miR-30c and speculate on the benefits of miR-30c over-expression in cancer therapy.

\section{The biogenesis of microRNA}

microRNAs (miRNAs or miRs) are endogenous non-coding single stranded small RNA of 20-24 nucleotides in eukaryotes. In 1993, Lee et al firstly reported a 22-nucleotides-long non-coding small RNA, LIN-4, which regulates the development of Caenorhabditiselegans[16]. Since then, a variety of microRNAs have been found. MicroRNAs play important roles in signal transduction, cell differentiation, proliferation, apoptosis, blood vessel formation and development, as well as inflammation and tumorigenesis in vivo. In eukaryotes, microRNAs are located in the non-coding region of genome, meanwhile, majority of microRNAs of human beings are located within introns or non-coding mRNA regions in genome with a portion of microRNAs located far from the transcribing regions[17].

During the biogenesis of microRNA, microRNAs are firstly transcribed into pri-microRNAs by RNA polymerase II[18], then pri-microRNAs are cleaved into pre-microRNAs of 70-80 nucleotides stem-loop by ribonuclease III-Drosha and double strand RNA binding protein-Pasha in nucleus[19]. Next, premicroRNAs were transported from nucleus to cytoplasm by GTP-dependent exportin5, where premicroRNAs were cleaved into microRNAs of 8-25 nucleotides mismatch-containing double-stranded RNA by ribonuclease III-Dicer. Double-stranded microRNAs were dissociated[20-22] and integrated into RNA-induced silencing complex (RISC), which forms the asymmetric RISC assembly to bind and regulate the target mRNA[23,24] via the specific binding to the $3^{\prime}$ UTR of target mRNAs (Figure 1).

In human genome, 30 percent of genes are regulated by the expression of microRNAs. It's shown that one microRNA can regulate multiple target mRNAs and one mRNA can be regulated coordinately by multiple microRNAs[25]. Future studies on microRNAs may reveal the complexity of eukaryotic genomes and the regulation mechanism of gene expression, and provide basis for disease diagnosis, treatment and prognosis.

\section{miR-30 family}

The miR-30 family is an important and complex family that plays vital roles in the function of miRNAs

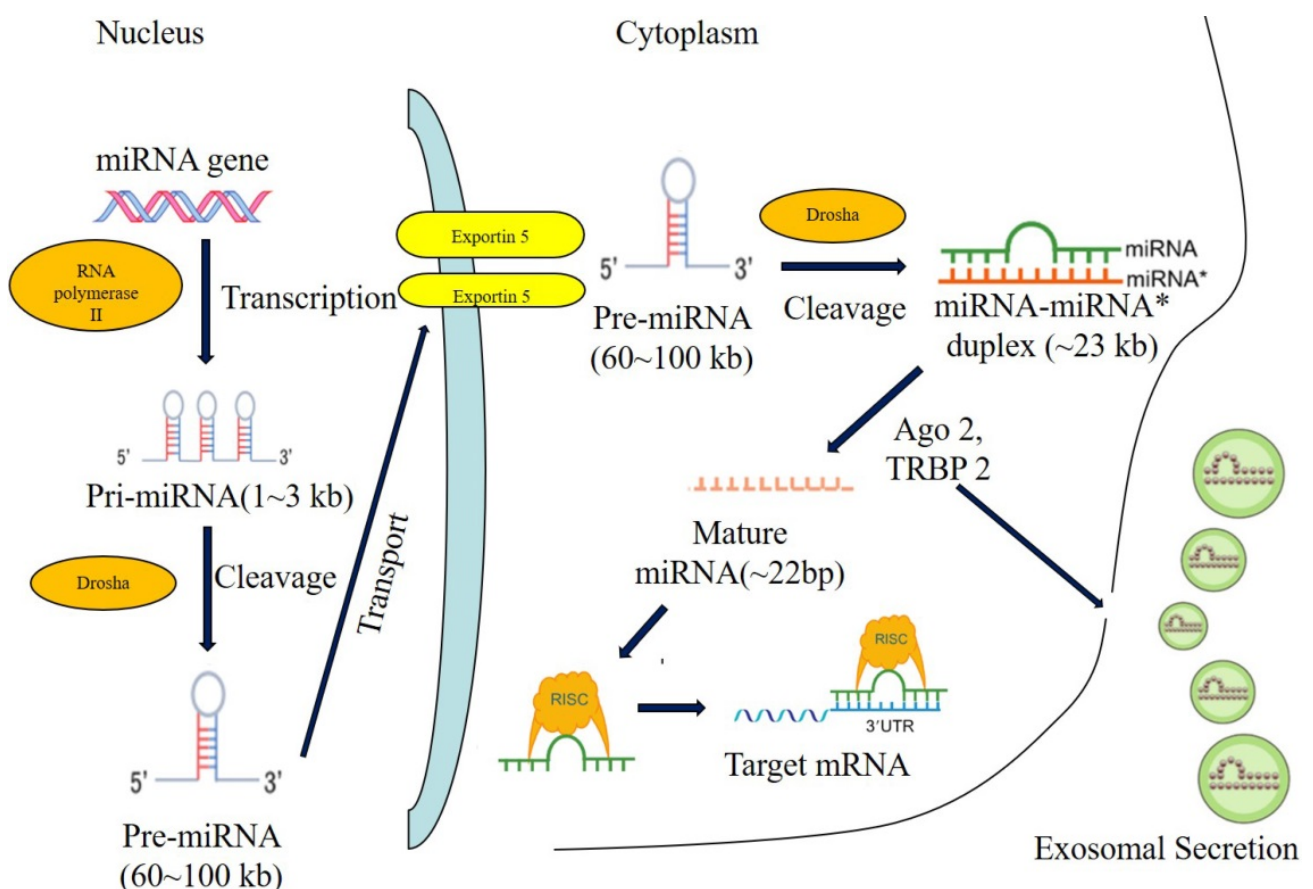

Figure 1. The biogenesis of miRNA. Note: Most miRNAs are transcribed by RNA polymerase II (RNA pol II) in the form of primary-miRNA (pri-miRNA), and then processed in the nucleus by Drosha andcleavaged to precursor miRNA (pre-miRNA). The pre-miRNA is further exported to the cytoplasm by exportin 5 and further cleavaged by a complex containing Ago 2 and TRBP 2. The functional strand of mature miRNA is incorporated into the RNA-induced silencing complex (RISC). As a component of this complex, the mature miRNA regulates the expression oftarget genes by specifically binding to these complementary sequences in the 3'UTR or coding regions of target mRNA, leading to the mRNA degradation or translational repression. Alternatively, the mature miRNA induce the translational activation by specifically binding to the 5 'UTR of target mRNA. In addition, miRNAs can be secreted through the exosomal pathway and regulate the gene expression of recipient cells. 
in mammalian and human beings. The miR-30 family contains 5 members and 6 different mature miRNA (miR-30a, -30b, -30c-1, -30c-2,-30d,-30e), which are encoded by 6 genes located on human chromosome 1 , 6 , and 8, respectively[26](Figure 2).

These miRNAs have the same seed sequence located near the $5^{\prime}$ end with different compensating sequences located near the $3^{\prime}$ end, which benefit the regulation process of different genes and pathways and sometimes lead to completely opposite behaviors. Consistently, a variety of physiological and pathological conditions in vivo are associated with differential expression of miR-30 family members via altering targeted gene expression[27].In human beings, miR30c (has-miRNA-30c or miRNA-30c) is encoded by genome 1 and 6 with miR-30c-1, belonging to the same group with miR-30e, encoded by genome 1 , and miR-30c-2 encoded by genome 6, respectively. In 2002, Lasgos-Quintana et al revealed that miR-30c or mmu-miR-30 was expressed in mouse heart and brain[28]. In 2004, miR-30c was found in TPAuntreated human myeloleukimia cells, demonstrating the existence of mature miR-30c in human cells for the first time[29]. Furthermore, Iwai et al revealed that human mature miR-30c was derived from pre-miR30c-2[30]. In addition, they also found that most mutated pre-miRNA were lost during the process of miRNA maturation, and only mature miR-30c-2 contains theses mutated sequences from pre-miR-30c2. The heterogeneity of miR-30c may alter target specificity, leading to the altered biological effects.

Recently, overwhelming evidences have suggested that the aberrant expression of miR-30 family is related with multiple cancers progression. As a member of miR-30 family, miR-30c is generally recognized as a multifunctional regulator of cell proliferation, differentiation, metabolism and apoptosis process, which is related with cancer metastasis and chemo-resistance in vivo. In this review, the associations between miR-30c dysregulation and cancer progression were investigate to speculate the benefits of miR-30c over-expression in the treatment of cancer (Figure 3).

\section{$\mathrm{miR}-30 \mathrm{c}$ and cancer progression}

The function of $\mathrm{miR}-30 \mathrm{c}$ in cancer progression

It has been found that miR-30c was low expressed in breast cancer[31], prostate cancer[32], colon cancer[33], medulloblastoma[34], as well as lung cancer[35], gastric cancer[36]and multiple myeloma [37], compared with these corresponding adjacent tissues. Increasing research shows miR-30c can be regulated or mutated by oncogenic transcription factors and cancer-associated genes. In 2008, Chang found the repression of multiple miRNA expression in Myc-induced B lymphocytes with miRNA chip and RNA blotting. The results showed that after $M y c$ induction, low expression of miRNA-30d/miRNA$30 \mathrm{~b}$ and miRNA-30e/miRNA-30c-1 were detected without the presence of miRNA-30a/miRNA-30c-2. Furthermore, the Myc repression byshRNA (short hairpin RNA) tranfection resulted in significant overexpression of multiple miRNAs, including miRNA30c. These above results demonstrated that expressions of multiple miRNAs, inclding miRNA-30c, were associated with the suppression of $M y c$-induced carcinogenesis[17]. Furthermore, the florescent labeling analysis results of breast cancer progression revealed that miR-30c binds $3^{\prime} \mathrm{UTP}$ of KRAS transcripts and silences the expression of KRAS mRNA and protein, which further inhibit the metastasis of breast cancer cells[38].

In 2012, Zhou found that miRNA-30c repressed the metabolism related gene-metabolism associated gene 1 (MTA1) in Ishikawa (estrogen receptor positive) and HEC-1-B (estrogen receptor negative) endometrial cancer cell lines, and played an inhibitory role in endometrial cancer progression[39]. They further found that over-expression of miR-30c in Ishikawa and HEC-1-B cell lines inhibited the cell proliferation, migration and invasion process. In 2014, Kong provided the direct evidence of miR-30c specific binding to the 3' UTP of MTA-1(Metastasis Associated 1), which further demonstrated that miR-30c was negatively related with endometrial cancer progression through MTA-1 inhibition [40].

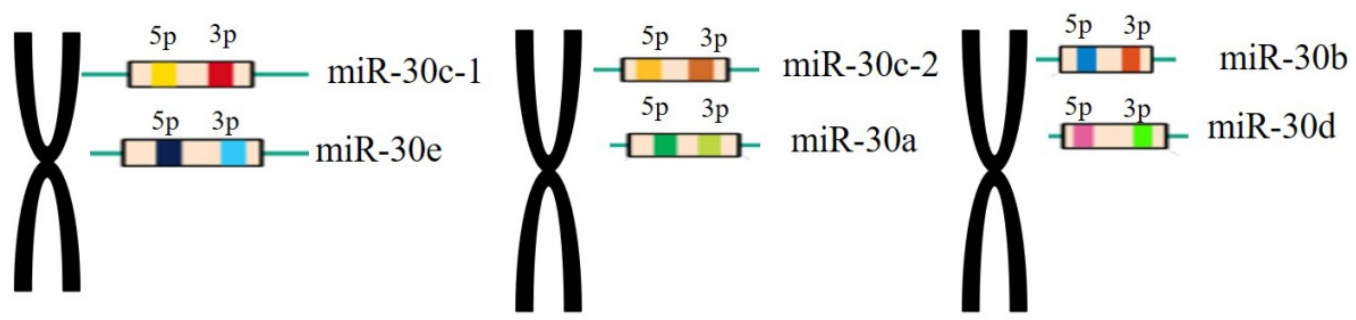

Chromosome 1

Chromosome 6

Chromosome 8

Figure 2. The miR-30 family members and their genomic locations. Note: miR-30c-1 and miR-30e are located in the chromosomes 1 withmiR-30c-2 and miR-30a located in the chromosomes 6 and miR-30b, miR-30d located in the chromosomes 8 , respectively. 


\section{A}

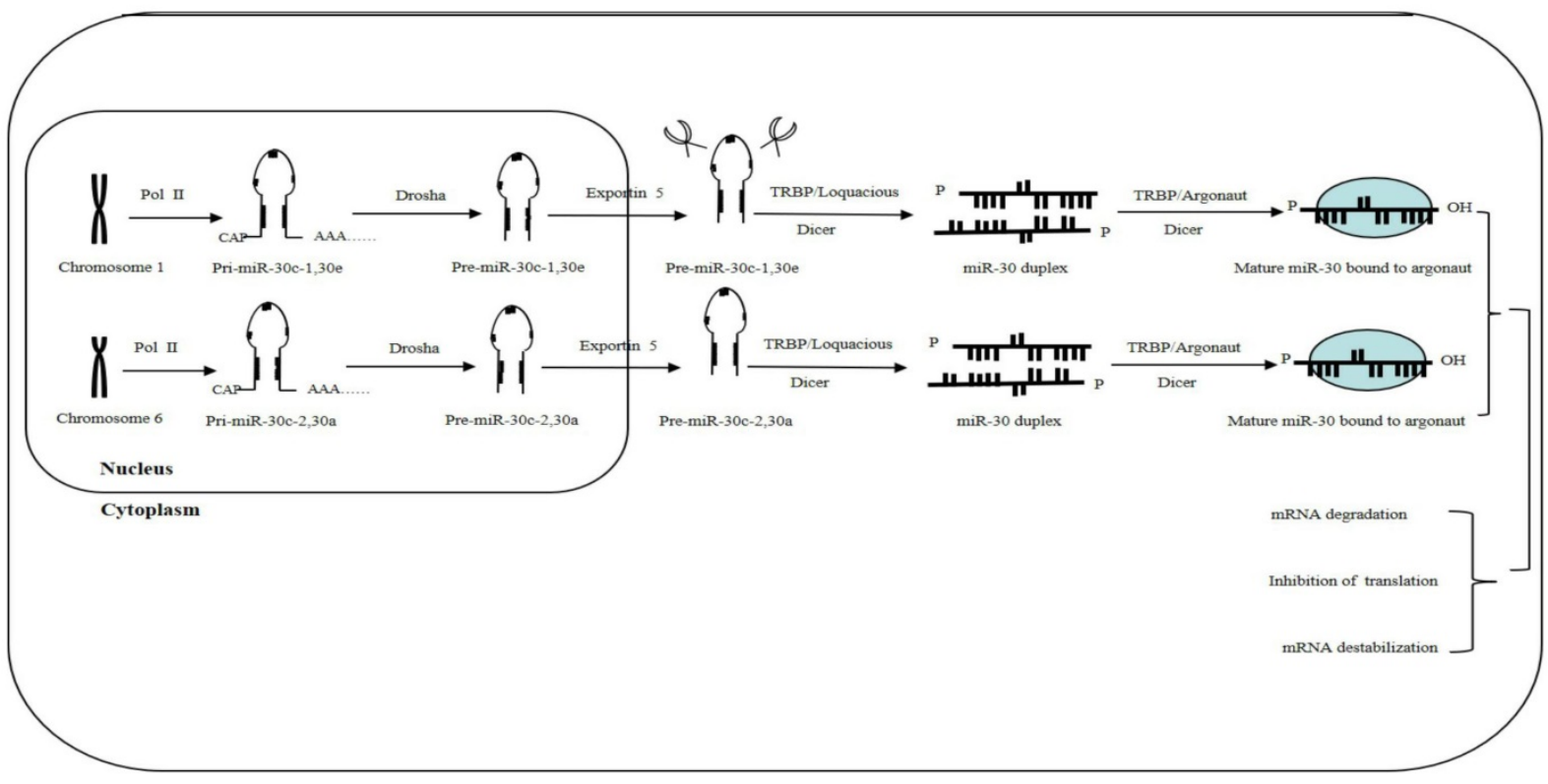

B

\section{Hsa-miR-30c-5p UGUAAACAUCCUACACUCUCAGC}

\section{Hsa-miR-30c-1-3p CUGGGAGAGGGUUGUUUACUCC}

\section{Hsa-miR-30c-2-3p CUGGGAGAAGGCUGUUUACUCU}

Figure 3. The biogenesis of miR-30c family. Note: (A) As important members of miRNA family, the miR-30c family, locatedon human chromosome 1 and 6 , contains 2 mature miRNA molecules (miR-30c-land miR-30c-2). (B) These mature miRNAs share a common seed sequence located near the 5' end with different compensatory sequences located near the 3' end. These different compensatory sequences allow miR-30c family members to target different genes and pathways, thus performing corresponding biological functions.

In 2013, it was reported that the expression levels of miR-30c were significantly increased in various cell lines, especially prostate and breast cancer cell lines, after sulphuretin treatment with decreased expression levels of cyclin D1 and cyclin D2. And the results also proved that miR-30c could specifically bind 3'UTR of cyclin D2[41].Another study showed that the expression of FHIT suppresses the epithelialmesenchymal transition (EMT) and metastasis of lung cancer through the modulation of miR-30c. The results further showed that miR-30c functions as a negative regulator of EMT and metastasis through directly targeting these mesenchymal markers, including vimentin, fibronectin, and these metastasisrelated genes, including MTDH and HMGA2, implying that miR-30c contributes to the regulation of EMT and cancer metastasis[42]. During the progres- sion of glioblastoma and lung cancer, miR-30c has been proved to be closely related with tumor necrosis factor (TNF)-related apoptosis-inducing ligand (TRAIL), which caused the apoptosis of cancer cells without killing the normal cells in vivo. The results of Quintavalle C's investigation showed that the expression levels of miRNAs, especially miR-30 b/c and miR-21, were significantly increased in the TRAIL-resistant glioma cells. In addition, the related regulation mechanism indicated that the specific bindings between miR-30 b/c ormiR-21 and $3^{\prime}$ UTR of caspase-3orTAp63 mRNAs regulated the expressions of downstream protein to produce resistance to TRAIL[43].In Wu W's research, they found that the migration and invasion abilities of SMMC-7721 and HepG2 liver cancer cells were negatively related with the expression levels of miR-30c through the specific 
binding between miR-30c and the $3^{\prime} \mathrm{UTR}$ of IER2, resulting in the decreasedIER2 protein expression level, which further affects the cell motilities[44]. In Gong's study, the miRNA expression profiles of natural killer (NK) cells conjugated with either anti-CD226 antibody (LeoA1) or control antibody were analyzed, and the results of miRNA array showed that 6 miRNAs were significantly decreased after LeoA1 treatments, including miR-30c and miR-30c-1. Meanwhile, the cytotoxicity of NK cells was enhanced through the inhibition of HMBOX1 via specific binding between miR-30c-1 and 3' UTR of HMBOX1, which leads to the increased expression levels of TNF- $\alpha$ (transmembrane TNF- $\alpha$, mTNF- $\alpha$ ) and repressed liver cancer progression[45]. Jia showed that the ovarian cell proliferation process induced by growth factors mediates a neutralizing response via significantly increased expression levels of miR-30c-2 which resulting in the decreased BCL9 (B-Cell CLL/ Lymphoma 9) expression levels and cell proliferation abilities during ovarian cancer progression[46]. In multiple myeloma cells, the proliferation, apoptosis, invasion, drug resistance and cancer stem cell formation abilities of cancer cells were significantly related with the specific bindings between miR-30c which maintains low expression level and the3'UTR of BCL9, and the following regulation of these downstream genes of Wnt/ $\beta$-catenin/BCL9 pathways [47]. See Table 1.

\section{The mutation of $\mathrm{miR}-30 \mathrm{c}$ and cancer progression}

Some studies showed that the polymorphism mutations ofmiR-30c played an oncogenic role during cancer progression. For instance, Fang suggested that SNPs rs71428439 (miR-149), rs2910164 (miR-146a), rs928508 (mir-30c-1) and rs629367 (let-7a-2) were associated with the lung cancer prevalence, polymorphisms of rs11614913 (miR-196a-2) and rs9280508 (miR-30c-1) significantly influenced the patients' response to platinum-based chemotherapy, which may serve as potential clinical biomarkers to predict lung cancer risk and platinum-based chemotherapy response[55].However, Yin group found that miR-196a2 rs11614913, miR-30c-1 rs928508, miR-608 rs4919510 and miR-27a rs895819 polymorphisms were not significantly associated with lung cancer risks in any models. The similar results were also found in lung adenocarcinoma patients[56].Hu demonstrated that the single nucleotide polymorphism (SNP) at rs928508(A/G) of miR-30c flanking region in lung cancer tissues regulated the transformation processes from pri-miR-30c to pre-miR-30c and mature miR-30c, but didn't affect the transcription process of pri-miR-30c, suggesting SNP may alter the process of miRNA maturation, expression and their targetbinding abilities, which further leads to lung cancer progression[57]. Another clinical research investigated the associations between SNP of miR-30c in gastric cancer tissues and the risk of cancer recurrence, and the results showed that the expression level of miR-30c was significantly higher in the gastric cancer tissues with the genotype of rs928508 AA than those with GG or AG/GG, and the pre-miR-30c AA genotype was positively associated with lymph node (LN) metastasis of gastric cancer patients[58].

Table 1. Role of miR-30c in human cancers

\begin{tabular}{|c|c|c|c|c|}
\hline Cancer & Expression & Target & Function\& Biomarker & Reference \\
\hline Myc-induced B cell lymphoma & & Unknown & Tumor-suppressing & 17 \\
\hline \multirow[t]{3}{*}{ Breast } & Decreased & KRAS & Inhibits metastasis & 48 \\
\hline & & VIM/TWF1 & Inhibits invasion;a potential sensitive chemotherapy biomarker & 38 \\
\hline & & HER/RACI & A potential tamoxifengood prognosis biomarker & 49 \\
\hline Colon & Decreased & ADAM19 & Inhibit cells proliferation, metastasis and invasion & 33 \\
\hline Bladder & Decreased & Unknown & Tumor-suppressing & 50 \\
\hline Medulloblastoma & Decreased & Unknown & Tumor-suppressing & 34 \\
\hline \multirow[t]{3}{*}{ Lung } & Decreased & Rab18 & Inhibited proliferation & 51 \\
\hline & & Vimentin, Fibronectin, and MTDH , HMGA2 & Inhibited metastasis & 52 \\
\hline & & Tyrosine kinases & A potential tyrosine kinase inhibitor sensitivity biomarker & 53 \\
\hline Endometrium & Decreased & MTA1 & $\begin{array}{l}\text { Tumor-suppressing, inhibited clles growth, migration and } \\
\text { invasion, }\end{array}$ & 39,40 \\
\hline \multirow[t]{2}{*}{ Prostate } & Decreased & CCND2 & $\begin{array}{l}\text { Tumor-suppressing and a potential Sulfuretin-sensitive } \\
\text { biomarker }\end{array}$ & 41 \\
\hline & & Possibly kRAS/MAPK & $\begin{array}{l}\text { Inhibited cell growth, invasion and metastasis.A potential } \\
\text { prognosis biomarker }\end{array}$ & \\
\hline \multirow[t]{2}{*}{ Liver } & Decreased & HMBOX1 & IFN-alfal and NK cell cytotoxicity increase & 45 \\
\hline & & IER2 & Inhibited cell motility & 44 \\
\hline Ovarian & Decreased & BCL9 & Inhibited cell proliferation & 46 \\
\hline large cell lymphoma (C-ALCL) & Increased & Unknown & $\begin{array}{l}\text { A different contribution to the pathogenesis of these } \\
\text { lymphomas. }\end{array}$ & 54 \\
\hline multiple myeloma & Decreased & BCL9 & $\begin{array}{l}\text { Influence tumor cell proliferation, apoptosis, transfer, drug } \\
\text { resistance and tumor stem cell formation }\end{array}$ & 37 \\
\hline
\end{tabular}




\section{The cellular, molecular and drug-} resistance mechanisms of MicroRNA-30c in cancer progression and the potential applications of MicroRNA-30c in clinics

\section{miR-30c as a potential biomarker for cancer patients}

In 2010, the miRNA screening results of 246 tamoxifen-treated breast cancer patients with positive estrogen expressions showed that only the expression levels of miRNA-30c, but not miRNA-30a-3p or miRNA-182, could be used as an independent prognostic biomarker for these breast cancer patients after tamoxifen treatment. The results also showed that these breast cancer patients with higher expression levels of miRNA-30c had better prognosis outcomes after tamoxifen treatment, while those patients with low expression levels of miRNA-30c suffered worse prognosis outcomes. Furthermore, they also found that the expression levels of miRNA-30c may be associated with HER and RACI pathways[59]. Gu analyzed the miRNA expression patterns between 41 NSCLC and 5 normal lung tissues with real-time RT-PCR, and the results showed that after the treatments of tyrosine kinase inhibitors (TKIs), as the first-line drug for NSCLC treatments, the expressions of both miR-30b and miR-30c were significantly down-regulated in lung cancer tissues. Further investigations demonstrated that patients with over-expression levels ofmiR-30b or miR-30c had better prognosis outcomes, suggesting miR-30b or miR-30c may be applied as potential prognostic biomarker for the TKIs treatment of NSCLC patients[53].

\section{The cellular, molecular and drug-resistance mechanisms of MiR-30c in cancer progression}

In 2013, Ling showed that the over-expression of miR-30c repressed the cell proliferation, invasion and metastasis abilities of prostate cancer via blocking the KRAS-MAPK pathway, meanwhile, the downregulated expression level of miR-30c predicts the early biochemical recurrence and worse prognosis of prostate cancer patients[60]. In Heinzelmann's research, 53 primary clear cell renal cell carcinoma tissues (ccRCC), 35 distant metastatic tissues from lung, bone, brain, and abdomen, as well as 17 normal kidney tissues were analyzed, and the results confirmed the significantly positive correlation between miR-30c expression and ccRCC metastasis, indicating that miR-30c could be used as a promising prognostic marker for early prediction of ccRCC metastasis and personalized cancer therapy[61]. Katzerke demonstrated that the transcription factor-
CCAAT enhancer binding protein alpha (C/EBPalpha) up-regulates the expression level of miR-30c in acute myeloid leukemia (AML) withNOTCH1as the direct target of miR-30c, indicating that miR-30c may be a novel biomarker and therapeutic targets of AML[62].

So far, miR-30c has been reported to be targeted and regulated by the transcription factors including Myc, HMBOX1 and C/EBP alpha with the target of miR-30cincludingMTA1， KRAS, BCL-9,Notchand cancer cell invasion related genes including TWF1, VIM and CCND2(Figure 4, 5,6).

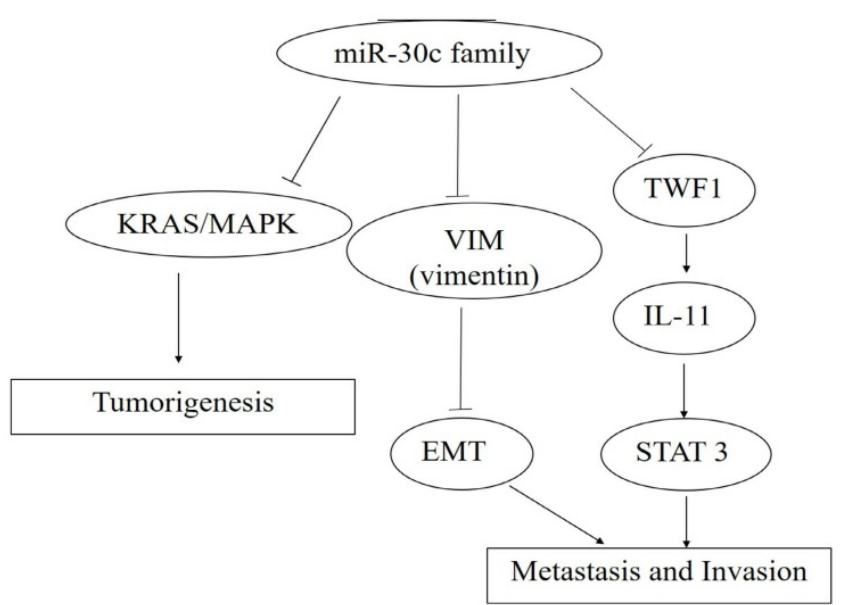

Figure 4. The roles of miR-30c family members in carcinogenesis, metastasis and invasion. Note: miR-30c promotes cancer progression through the activation of KRAS/MAPK signal pathway, and miR-30c family members act as caner suppressors by targeting TWFI (Twinfilin actin binding protein I)-IL-11-STAT3 pathway. Moreover, cancer metastasis and invasion were inhibited by the decreased EMT process caused by the down-expression leves ofMTDH(Metadherin) and VIM (Vimentin) via the specific bindings of miR-30c, respectively.

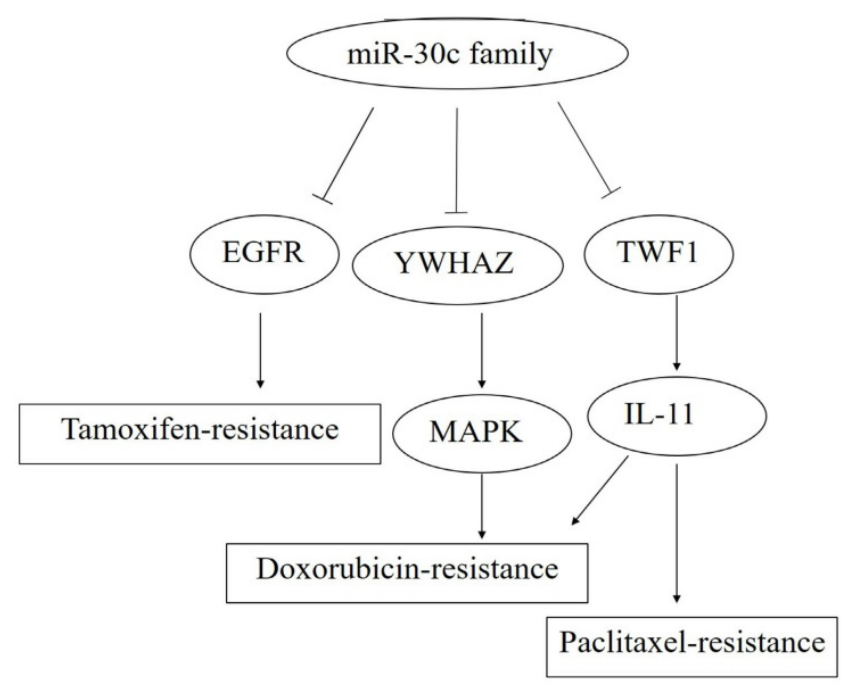

Figure 5. The roles of miR-30c family members in drug resistance. Note: miR-30c promotes the paclitaxel- and doxorubicin-resistance by blocking the TWF1-IL-11 pathway.Meanwhile,YWHAZ (Tyrosine 3-Monooxygenase/Tryptophan 5-Monooxygenase Activation Protein Zeta) was down-regulated by the specific binding with miR-30c, followed bythe activation of MAPK pathway andthe enhanced doxorubicin-resistance. In addition, miR-30c facilitates the tamoxifen resistance through the reduction of EGFR expression. 


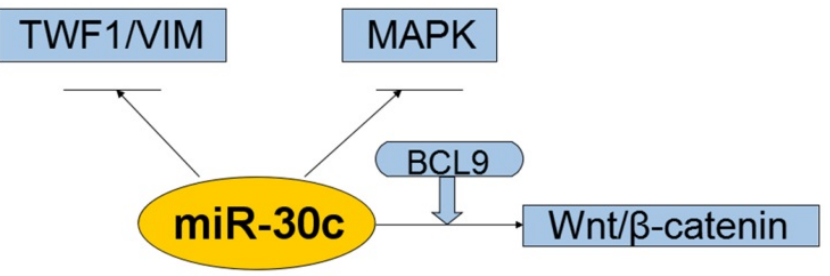

Figure 6. Interaction of the miR-30c with cellular pathways.miR-30c was down-regulated and exerts tumor-suppressing effects in cancers. All these suggest that miR-30c may be evolved to protect cells from carcinogenesis in most cases. So far it suggests that related pathways of miR-30c regulation cancers were kRAS/MAPK pathways, Wnt/b-catenin/BCL9 pathways, VIM/TWF1/IL-11 /PSTAT3pathways. Abbreviations: TWF1/VIM: cytoskeleton network genes encoding twinfilin I and vimentin; MAPK: mitogen-activated protein kinase; BCL9: B-cell CLL/lymphoma 9; miR-30c: microRNA 30c; Wnt: a group of secreted signaling molecules that mediate a variety of cellular processes.

In Song's research, miR-30c could be use as the potential biomarkers for the diagnosis and prognosis of prostate cancer patients and the detection of miRNAs including miR-30c is an effective way to predict patient's prognosis and evaluate the therapeutic efficacies [63].Liu found that miR-30c impedes glioblastoma cell proliferation and migration by targeting SOX9.In their study, they found that the expression levels of miR-30c were significantly downregulated in glioblastoma tissues and cancer cell lines. This research also found that after miR-30c overexpression, the majority of glioblastoma cells were arrested in $G_{0}$ phase and miR-30c over-expression suppressed the migration and invasion abilities of glioblastoma cells[64]. Ma reported miR-30c functions as a tumor suppressor via targeting SNAI1 in esophageal squamous cell carcinoma (ESCC). Their results showed that the expression level of miR-30c was significantly down-regulated in ESCC tissues and esophageal cancer cell lines. Due to the significant corrections between low expression level of miR-30c and worse prognosis outcomes of ESCC patients, miR-30c could be used as a promising biomarker and therapeutic target for ESCC in the future[65].miR-30c has complex roles during the regulation of physiologic and pathologic conditions. Current studies mostly focus on the function of one single miRNA in one specific cancer progression; however, the roles of miRNA vary according to cancer tissue types. Considering multiple targets of one single miRNA and multiple miRNAs targets of the same gene or mRNA, the future researches on miR-30c should use multiple cancer types with large scales to study the related network or complex pathways in order to understand the complex expression and regulation of miRNA-30c in cancer progression.

\section{The potential applications of miRNA-30c in clinics}

miR-30c can suggest clinical prognosis and novel tools for the therapy of various tumors. Busacca group showed the expression of miR-17-5p, miR-21, miR29a, miR-30c, miR-30e-5p, miR-106a, and miR-143 was significantly associated with the histopathological subtypes. Notably, the reduced expression of two miRNAs (miR-17-5p and miR-30c) correlated with better survival of patients with sarcomatoid subtype. They pointed at miRNAs as potential diagnostic and prognostic markers of mesothelioma, and suggests novel tools for the therapy of this malignancy [66].Quintavalle showed that high expression levels of miR-21 and $-30 \mathrm{~b} / \mathrm{c}$ are needed to maintain the TRAIL-resistant phenotype, thus making these miRs as promising therapeutic targets for TRAIL resistance in glioma [67]. Egeland suggested that seven microRNAs (miR-10a, miR-26, miR-30c, miR-126a, miR-210, miR-342 and miR-519a) played a role in tamoxifen resistance. Ingenuity Pathway Analysis (IPA) reveals that these seven microRNAs interact more readily with estrogen receptor (ER)-independent pathways than ER-related signaling pathways. Some of these pathways were targetable (e.g., PIK3CA), suggesting that microRNAs as biomarkers of endocrine resistance may have clinical value. Validation of the role of these candidate microRNAs in large prospective studies was warranted [68].

\section{Conclusions}

miR-30c maintains low expression levels in a variety of human cancer tissues and plays an anti-cancer role in cancer progression. However, more research is needed to confirm the functions of miR-30c in different tissues, organs and cancer progressions. Since these endogenous miRs regulate multiple pathways, it is necessary to investigate the various pathways and carefully over-interpret the beneficial effects of regulating one pathway. With the maturity of molecular biology and genetic engineering technology, the prognosis role of miR-30c will benefit the prevention, diagnosis and treatment of various diseases including cancer in the near future.

There are still many key issues that need to be further explained in the future, including (a) the specific roles and regulation mechanisms of different miR-30c family members in different cancer progressions, (b) the differences among miR-30c family members to perform subsequent targeted treatments, (c) the specific role of miR-30c family members in immune organ development and disease-related immune responses.

\section{Acknowledgements}

We are grateful to all participants in this study. This work was supported by a grant from the National Natural Science Foundation of China (\#81450047 and\#81660468), Inner Mongolia Natural 
Science Foundation Project of China (Grant Nos. 2017MS08 94), Inner Mongolia Autonomous Region Health and Family Planning Research Project of China (Grant Nos. 201702067, 201702098 and 201701078) ,Inner Mongolia Autonomous Region Science and Technology Plan Project (201802151)and the Inner Mongolia Medical University Youth Innovation Fund (No.YKD2016QNCX022). Our manuscript was translated and edited by the Editage.

\section{Author Contributions}

H.C., W.H. and X.S. designed the study, and wrote the review. X.S. conceived, designed the review. H.C., and J.L. edited and revised review. All authors discussed and approved the final version.

\section{Competing Interests}

The authors have declared that no competing interest exists.

\section{References}

1. Michlewski G, Cáceres JF. Post-transcriptional control of miRNA biogenesis. RNA. 2019; 25:1-16.

2. Quévillon Huberdeau M, Simard MJ. A guide to microRNA-mediated gene silencing. FEBS J. 2019;286:642-52.

3. Liu B, Li J, Cairns MJ. Identifying miRNAs, targets and functions. Brief Bioinform. 2014;15:1-19.

4. Guo T, Li J, Zhang L, Hou W, Wang R, Zhang J, et al. Multidimensional communication of microRNAs and long non-coding RNAs in lung cancer. J Cancer Res Clin Oncol. 2019;145:31-48.

5. Link A, Kupcinskas J. MicroRNAs as non-invasive diagnostic biomarkers for gastric cancer: Current insights and future perspectives. World J Gastroenterol. 2018;24:3313-29.

6. Pan JH, Zhou H, Zhao XX, Ding H, Li W, Qin L, et al. Role of exosomes and exosomal microRNAs in hepatocellular carcinoma: Potential in diagnosis and antitumour treatments (Review). Int J Mol Med. 2018;41:1809-16.

7. Stoicea N, Du A, Lakis DC, Tipton C, Arias-Morales CE, Bergese SD, et al. The MiRNA Journey from Theory to Practice as a CNS Biomarker. Front Genet. 2016;7:11.

8. Montironi R, Santoni M, Cimadamore A, Lopez-Beltran A, Cheng L. Editorial: Emerging Biomarkers in Genitourinary Tumors. Curr Drug Metab. 2019;9:326.

9. Korde A, Jin L, Zhang JG, Ramaswamy A, Hu B, Kolahian S, Guardela BJ, et al. Lung Endothelial MicroRNA-1 Regulates Tumor Growth and Angiogenesis. Am J Respir Crit Care Med. 2017;196:1443-55.

10. Slattery ML, Herrick JS, Mullany LE, Samowitz WS, Sevens JR, Sakoda L, et al. The co-regulatory networks of tumor suppressor genes, oncogenes, and miRNAs in colorectal cancer. Genes Chromosomes Cancer. 2017; 56: 769-87.

11. Sadik N, Cruz L, Gurtner A, Rodosthenous RS, Dusoswa SA, Ziegler O, et al. Extracellular RNAs: A New Awareness of Old Perspectives. Methods Mol Biol. 2018; 1740: 1-15.

12. Rizvi S, Khan SA, Hallemeier CL, Kelley RK, Gores GJ. Cholangiocarcinoma-evolving concepts and therapeutic strategies. Nature Reviews Clinical Oncology. 2018; 15:95-111.

13. Cheerla N, Gevaert O. MicroRNA based Pan-Cancer Diagnosis and Treatment Recommendation. BMC Bioinformatics. 2017;18:32.

14. Fan MJ, Zhong $\mathrm{YH}$, Shen $\mathrm{W}$, Yuan KF, Zhao GH, Zhang $\mathrm{Y}$, et al. MiR-30 suppresses lung cancer cell 95D epithelial mesenchymal transition and invasion through targeted regulating Snail. Eur Rev Med Pharmacol Sci. 2017;21:2642-49.

15. Kao CJ, Martiniez A, Shi XB, Yang J, Evans CP, Dobi A,et al. miR-30 as a tumor suppressor connects EGF/Src signal to ERG and EMT. Oncogene. 2014;33:2495-503.

16. Lee RC, Feinbaum RL, Ambros V. The C. elegans heterochronic gene lin-4 encodes small RNAs with antisense complementarity to lin-14. Cell. 1993;75:843-54

17 Chang TC, Yu D, Lee YS, Wentzel EA, Arking DE, West KM,et al. Widespread microRNA repression by Myc contributes to tumorigenesis. Nat Genet. 2008;40:43-50.

18. Lee Y, Kim M, Han J, Yeom KH, Lee S, Baek SH, et al. MicroRNA genes are transcribed by RNA polymerase II. Embo J. 2004;23:4051-60.

19. Lee Y, Ahn C, Han J, Choi H, Kim J, Yim J,et al. The nuclear RNase III Drosha initiates microRNA processing. Nature. 2003;425:415-9.

20. Bernstein E, Caudy AA, Hammond SM, Hannon GJ. Role for a bidentate ribonuclease in the initiation step of RNA interference. Nature. 2001;409:363-6.
21. Hutvágner G, McLachlan J, Pasquinelli AE, Bálint E, Tuschl T, Zamore PD. A cellular function for the RNA-interference enzyme Dicer in the maturation of the let-7 small temporal RNA. Science. 2001;293:834-8.

22. Ketting RF, Fischer SE, Bernstein E, Sijen T, Hannon GJ, Plasterk RH, et al. Dicer functions in RNA interference and in synthesis of small RNA involved in developmental timing in C. elegans. Genes Dev. 2001;15:2654-9.

23. Okamura K, Phillips MD, Tyler DM, Duan H, Chou YT, Lai EC, et al. The regulatory activity of microRNA* species has substantial influence on microRNA and 3' UTR evolution. Nat Struct Mol Biol. 2008; 15: 354-63.

24. Schwarz DS, Hutvágner G, Du T, Xu Z, Aronin N, Zamore PD. Asymmetry in the assembly of the RNAi enzyme complex. Cell. 2003;115:199-208.

25. Lewis BP, Burge CB, Bartel DP. Conserved seed pairing, often flanked by adenosines, indicates that thousands of human genes are microRNA targets. Cell. 2005;120:15-20.

26. Croset M, Pantano F, Kan CWS, Bonnelye E, Descotes F, Alix-Panabières C,et al. miRNA-30 Family Members Inhibit Breast Cancer Invasion, Osteomimicry, and Bone Destruction by Directly Targeting Multiple Bone Metastasis-Associated Genes. Cancer Res. 2018;78:5259-73.

27. Brennecke J, Stark A, Russell RB, Cohen SM. Principles of microRNA-target recognition. PLoS Biol. 2005; 3: e85.

28. Lagos-Quintana M, Rauhut R, Yalcin A, Meyer J, Lendeckel W, Tuschl T. Identification of tissue-specific microRNAs from mouse. Curr Biol. 2002;12:735-9.

29. Kasashima K, Nakamura Y, Kozu T. Altered expression profiles of microRNAs during TPA-induced differentiation of HL-60 cells. Biochem Biophys Res Commun. 2004;322:403-10.

30. Iwai N, Naraba H. Polymorphisms in human pre-miRNAs. Biochem Biophys Res Commun. 2005·331:1439-44.

31. Lai YH, Chen J, Wang XP, Wu YQ, Peng HT, Lin XH,et al. Collagen triple helix repeat containing-1 negatively regulated by microRNA-30c promotes cell proliferation and metastasis and indicates poor prognosis in breast cancer. J Exp Clin Cancer Res. 2017; 36: 92.

32. Ling $\mathrm{XH}, \mathrm{Han} Z \mathrm{D}, \mathrm{Xia} \mathrm{D}, \mathrm{He} H \mathrm{H}$, Jiang FN, Lin ZY, et al. MicroRNA-30c serves as an independent biochemical recurrence predictor and potential tumor suppressor for prostate cancer. Mol Biol Rep. 2014;41:2779-88.

33. Zhang Q, Yu L, Qin D, Huang R, Jiang X, Zou C, et al. Role of microRNA-30c targeting ADAM19 in colorectal cancer. PLoS One. 2015;10:e0120698.

34. Ferretti E, De Smaele E, Po A, Di Marcotullio L, Tosi E, Espinola MS, et al. MicroRNA profiling in human medulloblastoma. Int $\mathrm{J}$ Cancer. 2009;124:568-77.

35. McCann JV, Xiao L, Kim DJ, Khan OF, Kowalski PS, Anderson DG, et al. Endothelial miR-30c suppresses tumor growth via inhibition of TGF- $\beta$-induced Serpine1. J Clin Invest. 2019;130:1654-70.

36. $\mathrm{Mu} \mathrm{YP}, \mathrm{Su} X \mathrm{~L}$. Polymorphism in pre-miR-30c contributes to gastric cancer risk in a Chinese population. Med Oncol. 2011:29:1723-32.

37. Zhao JJ, Lin J, Zhu D, Wang X, Brooks D, Chen M, et al. miR-30-5p functions as a tumor suppressor and novel therapeutic tool by targeting the oncogenic Wnt/ $\beta$-catenin/BCL9 pathway. Cancer Res. 2014; 74: 1801-13.

38. Bockhorn J, Yee K, Chang YF, Prat A, Huo D, Nwachukwu C, et al. MicroRNA-30c targets cytoskeleton genes involved in breast cancer cell invasion. Breast Cancer Res Treat. 2013;137:373-82.

39. Zhou H, Xu X, Xun Q, Yu D, Ling J, Guo F, et al. microRNA-30c negatively regulates endometrial cancer cells by targeting metastasis-associated gene-1. Oncol Rep. 2012;27:807-12.

40. Kong X, Xu X, Yan Y, Guo F, Li J, Hu Y, et al. Estrogen regulates the tumour suppressor MiRNA-30c and its target gene, MTA-1, in endometrial cancer. PLoS One. 2014;9:e90810.

41. Poudel S, Song J, Jin EJ, Song K. Sulfuretin-induced miR-30C selectively downregulates cyclin D1 and D2 and triggers cell death in human cancer cell lines. Biochem Biophys Res Commun. 2013;431:572-8.

42. Suh SS, Yoo JY, Cui R, Kaur B, Huebner K, Lee TK, et al. FHIT Suppresses Epithelial-Mesenchymal Transition (EMT) and Metastasis in Lung Cancer through Modulation of MicroRNAs. PLoS Genet. 2014; 10: e1004652.

43. Quintavalle C, Donnarumma E, Iaboni M, Roscigno G, Garofalo M, Romano G,et al. Effect of miR-21 and miR-30b/c on TRAIL-induced apoptosis in glioma cells. Oncogene. 2013;32:4001-8.

44. Wu W, Zhang X, Liao Y, Zhang W, Cheng H, Deng Z,et al. miR-30c negatively regulates the migration and invasion by targeting the immediate early response protein 2 inSMMC-7721 and HepG2 cells. Am J Cancer Res. 2015;5:1435-46.

45. Gong J, Liu R, Zhuang R, Zhang Y, Fang L, Xu Z, et al. miR-30c-1* promotes natural killer cell cytotoxicity against human hepatoma cells by targeting the transcription factor HMBOX1. Cancer Sci. 2012;103:645-52.

46. Jia W, Eneh JO, Ratnaparkhe S, Altman MK, Murph MM. MicroRNA-30c-2* expressed in ovarian cancer cells suppresses growth factor-induced cellular proliferation and downregulates the oncogene BCL9. Mol Cancer Res. 2011;9:1732-45

47. Han W, Mu Y, Zhang Z, Su X. Expression of miR-30c and BCL-9 in gastric carcinoma tissues and their function in the development of gastric cancer. Oncol Lett. 2018;16:2416-26.

48. Tanic M, Yanowsky K, Rodriguez-Antona C, Andrés R, Márquez-Rodas I, Osorio A, et al. Deregulated miRNAs in hereditary breastcancer revealed a role for miR-30c in regulating KRAS oncogene. PLoS One. 2012;7:e38847

49. Rodríguez-González FG, Sieuwerts AM, Smid M, Look MP, Meijer-van Gelder ME, de Weerd V, et al. MicroRNA-30c expression level is an independent 
predictor of clinical benefit of endocrine therapy in advanced estrogen receptor positive breast cancer. Breast Cancer Res Treat. May 2011;127:43-51.

50. Wang G, Zhang H, He H, Tong W, Wang B, Liao G, et al. Up-regulation of microRNA in bladder tumor tissue is not common. Int Urol Nephrol. 2010;42:95-102.

51. Zhong K, Chen K, Han L, Li B. microRNA-30b/c inhibits non-small cell lung cancer cell proliferation by targeting Rab18. BMC Cancer. 2014,14:703.

52. Suh SS, Yoo JY, Cui R, Kaur B, Huebner K, Lee TK, et al. FHIT suppresses epithelial-mesenchymal transition (EMT) and metastasis in lung cancer through modulation of microRNAs. PLoS Genet. 2014; 10:e1004652.

53. Gu YF, Zhang H, Su D, Mo ML, Song P, Zhang F, et al. miR-30b and miR-30c expression predicted response to tyrosine kinase inhibitors as first line treatment in non-small cell lung cancer. Chin Med J (Engl). 2013; 126:4435-9.

54. Benner MF, Ballabio E, Kester MSV, Saunders NJ, Vermeer MH, Willemze R, et al. Primary cutaneous anaplastic large cell lymphoma shows a distinct miRNA expression profile and reveals differences from tumor-stage mycosis fungoides. Exp Dermatol. 2012;21:632-4.

55. Fang C, Li XP, Chen YX, Wu NY, Yin JY, Zhang W, et al. Functional miRNA variants affect lung cancer susceptibility and platinum-based chemotherapy response. J Thorac Dis. 2018;10:3329-40.

56. Yin Z, Cui Z, Ren Y, Xia L, Wang Q, Zhang Y, et al. Association between polymorphisms in pre-miRNA genes and risk of lung cancer in a Chinese non-smoking female population. Lung Cancer. 2016;94:15-21.

57. Hu Z, Shu Y, Chen Y, Chen J, Dong J, Liu Y, et al. Genetic polymorphisms in the precursor MicroRNA flanking region and non-small cell lung cancer survival. Am J Respir Crit Care Med. 2011;183:641-8.

58. $\mathrm{Mu} \mathrm{YP}, \mathrm{Su} X \mathrm{~L}$. Polymorphism in pre-miR-30c contributes to gastric cancer risk in a Chinese population. Med Oncol. 2012;29:1723-32.

59. Rodríguez-González FG, Sieuwerts AM, Smid M, Look MP, Meijer-van Gelder ME, de Weerd V, et al. MicroRNA-30c expression level is an independent predictor of clinical benefit of endocrine therapy in advanced estrogen receptor positive breast cancer. Breast Cancer Res Treat. 2011;127:43-51.

60. Ling XH, Han ZD, Xia D, He HC, Jiang FN, Lin ZY, et al. MicroRNA-30c serves as an independent biochemical recurrence predictor and potential tumor suppressor for prostate cancer. Mol Biol Rep. 2014;41:2779-88.

61. Heinzelmann J, Unrein A, Wickmann U, Baumgart S, Stapf M, Szendroi A, et al. MicroRNAs with prognostic potential for metastasis in clear cell renal cell carcinoma: a comparison of primary tumors and distant metastases. Ann Surg Oncol. 2014;21:1046-54.

62. Katzerke C, Madan V, Gerloff D. Notch1 during granulopoiesis and is downregulated in acute myeloid leukemia. Blood. 2013;122:2433-42.

63. Song CJ, Chen H, Chen LZ, Ru GM, Guo JJ, Ding QN. The potential of microRNAs as human prostate cancer biomarkers: A meta-analysis of related studies. J Cell Biochem. 2018;119:276327-86.

64. Liu S , Li X , Zhuang S. MiR-30c impedes glioblastoma cell proliferation and migration by targeting SOX9. Oncol Res. 2019;27:165-71.

65. Ma T, Zhao Y, Lu Q, Lu Y, Liu Z, Xue T, et al. MicroRNA-30c functions as a tumor suppressor via targeting SNAI1 in esophageal squamous cell carcinoma. Biomed Pharmacother. 2018;98:680-6.

66. Busacca S, Germano S, De Cecco L, Rinaldi M, Comoglio F, Favero F, et al. MicroRNA signature of malignant mesothelioma with potential diagnostic and prognostic implications. Am J Respir Cell Mol Biol. 2010;42:312-9.

67. Quintavalle C, Donnarumma E, Iaboni M, Roscigno G, Garofalo M, Romano $\mathrm{G}$, et al. Effect of miR-21 and miR-30b/c on TRAIL-induced apoptosis in glioma cells. Oncogene. 2013;32:4001-8.

68. Egeland NG, Lunde S, Jonsdottir K, Lende TH, Cronin-Fenton D, Gilje B,et al. The Role of MicroRNAs as Predictors of Response to Tamoxifen Treatment in Breast Cancer Patients. Int J Mol Sci. 2015;16:24243-75. 